\title{
Screening effects in superconductors
}

\author{
M. Capezzali ${ }^{\mathrm{a}, *}$, D. Ariosa ${ }^{\mathrm{b}}$, H. Beck ${ }^{\mathrm{a}}$ \\ a Physics Department, University of Neuchâtel, Breguet 1,2000 Neuchâtel Switzerland \\ b IBM Research Division, Säumerstr. 4,8803 Rüschlikon (ZH), Switzerland
}

\begin{abstract}
The partition function of the Hubbard model with local attraction and long-range Coulomb repulsion between electrons is written as a functional integral with an action $A$ involving a pairing field $\Delta$ and a local potential $V$. After integration over $V$ and over fluctuations in $|\Delta|^{2}$, the final form of $A$ involves a Josephson coupling between the local phases of $\Delta$ and a "kinetic energy" term, representing the screened Coulomb interaction between charge fluctuations. The competition between Josephson coupling and charging energy allows to understand the relation between $T_{\mathrm{C}}$ and composition in high- $T_{\mathrm{C}}$ materials, in particular superlattices, alloys and bulk systems of low doping.
\end{abstract}

Keywords: Coulomb interaction; Phase diagram; Phase dynamics

We start with a Hamiltonian describing charge carriers (electrons or holes) on a lattice, subject to an on-site attraction, $-U$, and the long-range Coulomb repulsion, $e^{2} V_{\mathrm{C}}$, acting on particles on different sites:

$H=H_{0}+H_{U}+H_{\mathrm{C}}$,

$H_{0}=\sum_{\left\langle l, l^{\prime}\right\rangle} \sum_{\sigma} t c_{l, \sigma}^{+} c_{l^{\prime}, \sigma}-\mu \sum_{l} N_{l}$,

$H_{U}=-\frac{U}{2} \sum_{l, \sigma} c_{l, \sigma}^{+} c_{l, \sigma} c_{l,-\sigma}^{+} c_{l,-\sigma}$

$H_{\mathrm{C}}=\frac{1}{2} \sum_{l \neq l^{\prime}}\left(N_{l}-n_{0}\right) e^{2} V_{\mathrm{C}}\left(l, l^{\prime}\right)\left(N_{l^{\prime}}-n_{0}\right)$

Here $c_{l, \sigma}^{+}\left(c_{l, \sigma}\right)$ are creation (annihilation) operators for charge carriers with spin $\sigma$ at lattice site $l,\left\langle l, l^{\prime}\right\rangle$ denotes pairs of nearest-neighbor sites, $\mu$ is the chemical potential, $n_{0}$ the background, neutralizing

* Corresponding author. the density of charge carriers, and $N_{l}=\sum_{\sigma} c_{l, \sigma}^{+} c_{l, \sigma}$. The partition function can be written as a functional integral by means of two successive Stratonovich-Hubbard transformations $[1,2]$, decoupling the two interaction terms in $H$ with the help of a complex field $\Delta$ and a real field $V$ :

$$
\begin{aligned}
Z= & \operatorname{Tr} \mathrm{e}^{-\beta H}=\int D^{2} \Delta \int D V \operatorname{Tr} \mathrm{e}^{-\beta H_{0}} \\
& \times T \exp \left\{-i \int_{0}^{-i \beta} \mathrm{d} \tau[\tilde{H}(\Delta, V, \tau)+\varepsilon(\Delta, V, \tau)\},\right.
\end{aligned}
$$

$$
\begin{aligned}
\tilde{H}(\Delta, V, \tau)= & \sum_{l}\left[\Delta^{*}(l, \tau) c_{l, \uparrow} c_{l, \downarrow}+\right.\text { h.c. } \\
& \left.+i V(l, \tau)\left(N_{l}(\tau)-n_{0}\right)\right], \\
\varepsilon(\Delta, V, \tau)= & \frac{1}{U} \sum_{l}\left|\Delta(l, \tau)^{2}\right|+\frac{1}{2 e^{2}} \\
& \times \sum_{l \neq l^{\prime}} V(l, \tau) V_{\mathrm{c}}^{-1}\left(l, l^{\prime}\right) V\left(l^{\prime}, \tau\right) .
\end{aligned}
$$


We then evaluate the trace over the electronic degrees of freedom

$$
\begin{aligned}
& \operatorname{Tr} \mathrm{e}^{-\beta H_{0}} T \exp \left\{-i \int_{0}^{-i \beta} \mathrm{d} \tau[\tilde{H}(\Delta, V, \tau)\right. \\
& +\varepsilon(\Delta, V, \tau)]\} \equiv \exp \left\{-i \int_{0}^{i \beta} \mathrm{d} \tau F(\Delta, V, \tau)\right\}
\end{aligned}
$$

and we expand the "free energy" $F$ up to fourth order in $\Delta$ and to second order in $V$ and up to leading terms in space and time gradients of the two fields:

$$
\begin{aligned}
& F=F_{0}+ F_{\Delta, V} \\
& F_{\Delta, V}(\tau)=\sum_{l}\left[a|\Delta(l, \tau)|^{2}-i d \Delta^{*}(l, \tau)\right. \\
&\left.\times\left(\frac{\partial}{\partial \tau}-2 V(l, \tau)\right) \Delta(l, \tau)\right] \\
&+c \sum_{\left\langle l, l^{\prime}\right\rangle}\left|\Delta(l, \tau)-\Delta\left(l^{\prime}, \tau\right)\right|^{2} \\
&+i \sum_{l} V(l, \tau)\left(\left\langle N_{l}\right\rangle-n_{0}\right) \\
&+\frac{1}{2 e^{2}} \sum_{l, l^{\prime}} V(l, \tau) V_{\mathrm{SC}}^{-1}\left(l, l^{\prime}\right) V\left(l^{\prime}, \tau\right) \\
&+b \sum_{l}|\Delta(l, \tau)|^{4} .
\end{aligned}
$$

Here the coefficients $a, b, c, d$, are related to the free-electron particle-particle propagator [1-5], $F_{0}$ is the free-electron contribution, and $V_{\mathrm{SC}}^{-1}\left(l, l^{\prime}\right)=V_{\mathrm{C}}^{-1}\left(l, l^{\prime}\right)+\chi_{0}\left(l, l^{\prime}\right)$ is the screened Coulomb potential (approximated by its static limit) with $\chi_{0}\left(l, l^{\prime}\right)$ being the electronic polarizability. Integrating over the electric potential $V$ yields

$$
\begin{aligned}
F_{\Delta}(\tau)= & \sum_{l}\left[a|\Delta(l, \tau)|^{2}+b|\Delta(l, \tau)|^{4}\right. \\
& \left.-\mathrm{i} d \Delta^{*}(l, \tau) \frac{\partial \Delta(l, \tau)}{\partial \tau}\right] \\
& +c \sum_{\left\langle l, l^{\prime}\right\rangle}\left|\Delta(l, \tau)-\Delta\left(l^{\prime}, \tau\right)\right|^{2} \\
& +\frac{1}{2 e^{2}} \sum_{l, l^{\prime}} \rho(l, \tau) V_{\mathrm{SC}}\left(l, l^{\prime}\right) \rho\left(l^{\prime}, \tau\right),
\end{aligned}
$$

where $\rho(l, \tau)=2 \mathrm{~d}|\Delta(l, \tau)|^{2}+\left\langle N_{l}\right\rangle-n_{0}$ represents charge density fluctuations. Next we introduce am- plitude and phase of $\Delta$. In the following, we assume to be in a relatively strong coupling regime $(t \ll U)$ in which fermions bind into on-site singlet pairs at a temperature of the order of the mean field transition temperature $T_{\mathrm{mf}}$, well above the superconducting phase transition, the latter being finally triggered by the onset of phase order [6-8]. Below $T_{\mathrm{mf}}, a<0$, so that the average amplitude has a non-vanishing mean value $\Delta_{0}$ given by $a+2 b \Delta_{0}^{2}=0$. Charge neutrality implies $2 d \Delta_{0}^{2}+$ $\left\langle N_{l}\right\rangle-n_{0}=0$ and, for $t \ll U[1,3-5,9]$ :

$c=\frac{2 t^{2}}{U^{3}}, \quad d=\frac{1}{U^{2}}$,

$\Delta_{0}^{2}=\frac{1}{4} n_{0}\left(2-n_{0}\right) U^{2} \approx \frac{1}{2} n_{0} U^{2}$ for $n_{0} \ll 1$.

Splitting the number of pairs at a given site into $|\Delta(l, \tau)|^{2}=\Delta_{0}^{2}+\frac{1}{2} U^{2} \delta n_{\mathrm{p}}(l, \tau)$, we integrate over $\delta n_{\mathrm{p}}(l, \tau)$ which (neglecting gradient terms) yields a free energy functional for phase fluctuations only:

$$
\begin{aligned}
F_{\Theta}(\tau)= & J \sum_{\left\langle l, l^{\prime}\right\rangle}\left[1-\cos \left(\Theta(l, \tau)-\Theta\left(l^{\prime}, \tau\right)\right)\right] \\
& +\frac{n_{0}}{2} \sum_{l} \frac{\partial \Theta(l, \tau)}{\partial \tau}-\frac{1}{2} \sum_{l, l^{\prime}} \frac{\partial \Theta(l, \tau)}{\partial \tau} \\
& \times \frac{1}{(2 e)^{2}} W^{-1}\left(l, l^{\prime}\right) \frac{\partial \Theta\left(l^{\prime}, \tau\right)}{\partial \tau}
\end{aligned}
$$

with the Josephson coupling $[1,9] J=2 c \Delta_{0}^{2}=$ $2 n_{0}\left(t^{2} / U\right)$, and

$W\left(l, l^{\prime}\right)= \begin{cases}V_{\mathrm{SC}}\left(l, l^{\prime}\right), & l \neq l^{\prime}, \\ (2 e)^{2} b / d^{2}, & l=l^{\prime}\end{cases}$

Our expansion of $F$ in powers of $\Delta$ has yielded the on-site repulsion $(2 e)^{2} b / d^{2}$ between pairs. However, due to the exclusion principle, two pairs cannot really sit on the same lattice site. Thus, in the following, we exclude $l=l^{\prime}$ in the last term of (8a). By going from the "phase velocities" $(\partial \Theta(l, \tau) / \partial \tau)$ to the conjugate momenta $p(l, \tau)$ we end up with the partition function of the Hamiltonian [10]

$$
\begin{aligned}
H= & \frac{1}{2} \sum_{l, l^{\prime}}\left(p(l)-\frac{n_{0}}{2}\right)\left[(2 \mathrm{e})^{2} W\left(l, l^{\prime}\right)\right] \\
& \times\left(p\left(l^{\prime}\right)-\frac{n_{0}}{2}\right)+J \sum_{\left\langle l, l^{\prime}\right\rangle}\left[1-\cos \left(\Theta(l)-\Theta\left(l^{\prime}\right)\right)\right] .
\end{aligned}
$$


Hamiltonian (9) describes the relevant physics of short coherence length superconductors in terms of Josephson coupled spatial phase variations and "charge fluctuations" coupled by the screened Coulomb interaction. It is also the "phase-only" representation of the Hamiltonian of interacting bosons [10-12]. Its critical behavior, in particular the influence of the "background", $-\frac{1}{2} n_{0}$, has been studied in these references. Here, we apply expression (9) to calculate the transition temperature of strongly anisotropic superconductors, such as superlattices and bulk systems in the underdoped regime [13]. We make the following approximations: (i) $H$ is restricted to one superconducting layer; (ii) the screened Coulomb interaction, which takes into account the electric coupling between layers, is modelled by a Yukawa-form, with a Thomas-Fermi screening length $\lambda_{\mathrm{TF}}$, depending on the density $n_{0}$ of charge carriers according to the well-known formula

$\lambda_{\mathrm{TF}}^{-1}=\frac{1}{2 \pi} \frac{2.95}{\sqrt{r_{\mathrm{s}} / a_{0}}}\left[\AA^{-1}\right]$,

where $r_{\mathrm{s}}=\left(3 /\left(4 \pi n_{0}\right)\right)^{1 / 3}$ and $a_{0}$ is the Bohr radius; (iii) considering only $n_{0} \ll 1$, the "background shift", $-\frac{1}{2} n_{0}$, in the first term of (9) is neglected. We make connection with previous work $[14,15]$ by mapping (9) onto a "capacity model":

$\frac{1}{2} \sum_{l, l^{\prime}} p(l)\left[(2 e)^{2} W\left(l, l^{\prime}\right)\right] p\left(l^{\prime}\right) \approx \frac{(2 e)^{2}}{2 C} \sum_{l} p(l)^{2}$,

with

$\frac{1}{2 C}=\frac{1}{e^{2}} \sum_{l=0} W(l, 0)=\frac{2 \pi \lambda_{\mathrm{TF}}}{\varepsilon a_{\mathrm{L}}^{2}} \mathrm{e}^{-a_{\mathrm{L}} / \lambda_{\mathrm{TF}}}$.

This is the $X Y$-model with kinetic energy $[14,15]$, $\varepsilon$ being the dielectric constant of the interlayer material and $a_{\mathrm{L}}$ the lattice constant. In Ref. [14] the critical temperature in two dimensions was evaluated in the "self-consistent harmonic approximation" (SCHA) which gives results in good agreement with Monte Carlo simulations [16]. A good overall fit of the numerical SCHA result is [17] $T_{\mathrm{C}}(\alpha) \approx T_{\mathrm{C}}(0) \sqrt{1-\alpha / \alpha_{\mathrm{C}}}$ where $\alpha=(2 e)^{2} /(2 C J)$ is the ratio between charging and Josephson energy. When $\alpha$ approches $\alpha_{C}=6.2, T_{C}$ goes to zero. This approach has been successfully applied $[14,15]$ to calculating the superconducting transition temperature for superlattices and for alloys by determining the appropriate effective capacity through electrostatic considerations.

We finally use Hamiltonian (9) to find $T_{\mathrm{C}}$ as a function of doping for high- $T_{\mathrm{C}}$ superconductors in the underdoped regime. For $\mathrm{YBa}_{2} \mathrm{Cu}_{3} \mathrm{O}_{7}$, our "capacity model" has allowed to fit the $T_{\mathrm{C}}$ variation of both, superlattices and alloys, in a coherent way, using SCHA $[14,15]$ for $J=120 \mathrm{~K}$ at optimal doping $\left(n_{0} \approx 0.16\right.$ of holes per cell [13]). Below optimal doping, the ratio $\alpha$ increases when the number $n_{0}$ of charge carriers is reduced: the $n_{0}$ dependence of $J$ is given following Eq. (8a) and $C$ varies with $n_{0}$ through the screening length $\lambda_{\text {TF }}$. Using the above expression for $T_{\mathrm{C}}(\alpha)$, with dopingdependent $J$ and $C$, we then find that $T_{\mathrm{C}}$ should be zero for $n_{0} \approx 0.07$, in good agreement with the measured phase diagram [13]. This shows that the phase boundary in the underdoped regime can be understood in terms of Bose-Einstein condensation of preformed pairs, $T_{\mathrm{C}}$ being suppressed by phase fluctuations when the minimum doping is approached.

In summary, starting from the attractive Hubbard model with long-range Coulomb repulsion, we have given a microscopic derivation of a description of short coherence length superconductors in terms of the superconducting phase, the Hamiltonian for which includes a "charging energy" and a Josephson coupling. This is a microscopic justification of such a Hamiltonian, which has been used previously $[14,15]$ in calculating $T_{\mathrm{C}}$ for superlattices and alloys and also allows understanding the phase boundary of bulk oxides in the underdoped regime.

This work was supported by the Swiss National Science Foundation. We thank T. Schneider and M.H. Pedersen for valuable discussions.

\section{References}

[1] T.K. Kopec and H. Umezawa, Phys. Rev. B 47 (1993) 8923.

[2] V. Ambegaokar, U. Eckern and G. Schön, Phys. Rev. Lett. 46 (1982) 211.

[3] M. Drechsler and W. Zwerger, Ann. Phys. 1 (1992) 15. 
[4] M. Randeria, in: "Bose-Einstein Condensation", eds. A. Griffin, D. Snoke and S.S. Tringari (Cambridge University Press, Cambridge, 1994).

[5] M.H. Pedersen and H. Beck, unpublished.

[6] V.J. Emery and S.A. Kivelson, Nature 374 (1995) 434, Phys. Rev. Lett. 74 (1995) 3253.

[7] S. Doniach and M. Inui, Phys. Rev. B 41 (1990) 6668.

[8] E. Roddick and D. Stroud, Phys. Rev. Lett. 74 (1995) 1430.

[9] J.J. Vicente Alvarez and C.A. Balseiro, Solid State Commun. 98 (1996) 313.

[10] M.P.A. Fisher and G. Grinstein, Phys. Rev. Lett. 60 (1988) 208.
[11] Min-Chul Cha and S.M. Girvin, Phys. Rev. B 49 (1994) 9794.

[12] M.P.A. Fisher, P.B. Weichmann, G. Grinstein and D.S. Fisher, Phys. Rev. B 40 (1989) 546; A. van Otterlo, K.H Wagenblast, R. Baltin, C. Bruder, R. Fazio and G. Schön, Phys. Rev. B 52 (1995) 16176.

[13] J.L. Tallon and N.E. Flower, Physica C 204 (1993) 237.

[14] D. Ariosa and H. Beck, Phys Rev B 43 (1991) 344.

[15] D. Ariosa, T. Luthy, V. Tsaneva, B. Jeanneret, H. Beck and P.Martinoli, Physica B 194-196 (1994) 2371.

[16] L. Jacobs, J.V. Jose, M.A. Novotny and A.M. Goldman, Phys. Rev. B 38 (1988) 4562.

[17] D. Ariosa, J. Perret, H. Beck and P. Martinoli, in preparation. 